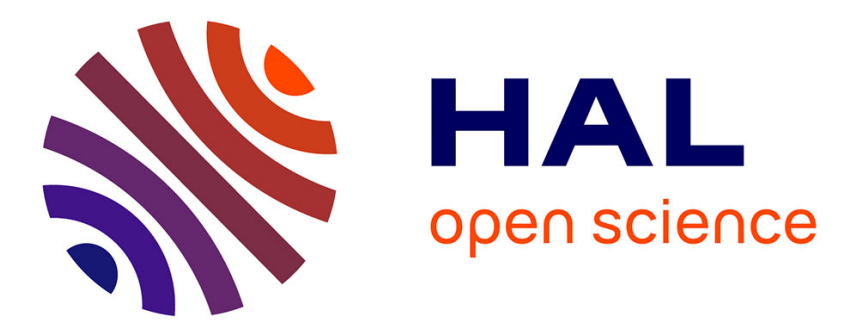

\title{
Quito face à un risque d'origine naturelle: les laves torrentielles
}

Jean Louis Perrin, Benoît Fourcade, Jérôme Poulenard, Didier Richard, Alexis Sierra

\section{- To cite this version:}

Jean Louis Perrin, Benoît Fourcade, Jérôme Poulenard, Didier Richard, Alexis Sierra. Quito face à un risque d'origine naturelle: les laves torrentielles. Revue de Géographie Alpine / Journal of Alpine Research, 2000, 88 (2), pp. 43-47. 10.3406/rga.2000.2990 . halsde-00799015

\section{HAL Id: halsde-00799015 https://hal.science/halsde-00799015}

Submitted on 8 Feb 2021

HAL is a multi-disciplinary open access archive for the deposit and dissemination of scientific research documents, whether they are published or not. The documents may come from teaching and research institutions in France or abroad, or from public or private research centers.
L'archive ouverte pluridisciplinaire HAL, est destinée au dépôt et à la diffusion de documents scientifiques de niveau recherche, publiés ou non, émanant des établissements d'enseignement et de recherche français ou étrangers, des laboratoires publics ou privés.

\section{(c)(1)}

Distributed under a Creative Commons Attribution| 4.0 International License 


\title{
Quito face à un risque d'origine naturelle : les laves torrentielles M. Jean-Louis Perrin, M. Benoît Fourcade, Monsieur Jerôme Poulenard, D. Richard, A. Sierra
}

\begin{abstract}
Abstract : The 31st of March 1997 a debris flow seriously affected the quebrada La Comunidad and. Morpho-hydro-climatic studies done after this event showed that extreme rainfall storms was the main factor to explain these phenomenons, it is evident that the morphology of the catchment, and particularly the longitudinal profile, played a fundamental part in the generation of debris flows. These events seem preferentially take place in the small catchments, therefore they can be considered as more dangerous than the big ones for the town suburban zones.
\end{abstract}

\section{Résumé}

Résumé : Le 31 mars 1997, une lave torrentielle affectait gravement la quebmda La Comunidad. Des études morpho-hydroclimatiques menées à la suite de cet accident ont montré que si l'événement pluvieux de fréquence rare était l'un des facteurs principaux d'explication du phénomène, il apparaissait clairement que la morphologie même des quebradas, et en particulier leur profil en long, pouvait jouer un rôle fondamental dans la génération de ceux-ci. De même, il semble que les petites quebradas soient plus sujettes à ce type d'événements, les rendant ainsi plus dangereuses que les grandes pour les quartiers situés en périphérie de la ville.

\section{Citer ce document / Cite this document :}

Perrin Jean-Louis, Fourcade Benoît, Poulenard Jerôme, Richard D., Sierra A. Quito face à un risque d'origine naturelle : les laves torrentielles. In: Revue de géographie alpine, tome 88, n², 2000. pp. 43-57;

doi : https://doi.org/10.3406/rga.2000.2990

https://www.persee.fr/doc/rga_0035-1121_2000_num_88_2_2990

Fichier pdf généré le 22/04/2018 


\section{Quito face à un risque d'origine naturelle : les laves torrentielles}

Perrin J.-L., Fourcade B.

IRD - Centre de Montpellier - 911, av. Agropolis - BP 5045 - F30032 Montpellier cedex

Poulenard J.

CNRS - Centre de Pédologie Biologique -17, rue Notre-Dame des Pauvres - F54501 Vandœuvre-les-Nancy

Richard D.

Cemagref - Groupement de Grenoble - 2, rue de la Papeterie - BP 76 - F38402 Saint-Martin-d'Hères cedex

Sierra A.

IRD - Centre de Bondy - 32, av. Henri Varagnat - F93143 Bondy cedex

\section{Introduction}

La ville de Quito occupe un gradin tectonique orienté sud-nord situé en bordure du sillon interandin, à une altitude moyenne de $2800 \mathrm{~m}$ (Fig. 1). Dominée par les volcans Pichincha (4627 m) et Atacazo (4 $455 \mathrm{~m}$ ) à l'ouest, elle est limitée à l'est par un décrochement de faille surplombant les vallées de Tumbaco et de Los Chillos $(2500 \mathrm{~m})$.

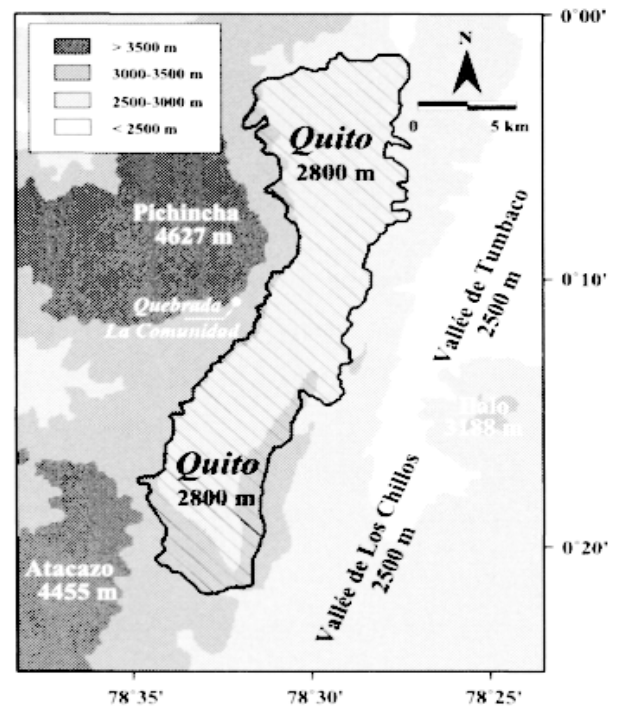

Fig. 1 : Situation générale

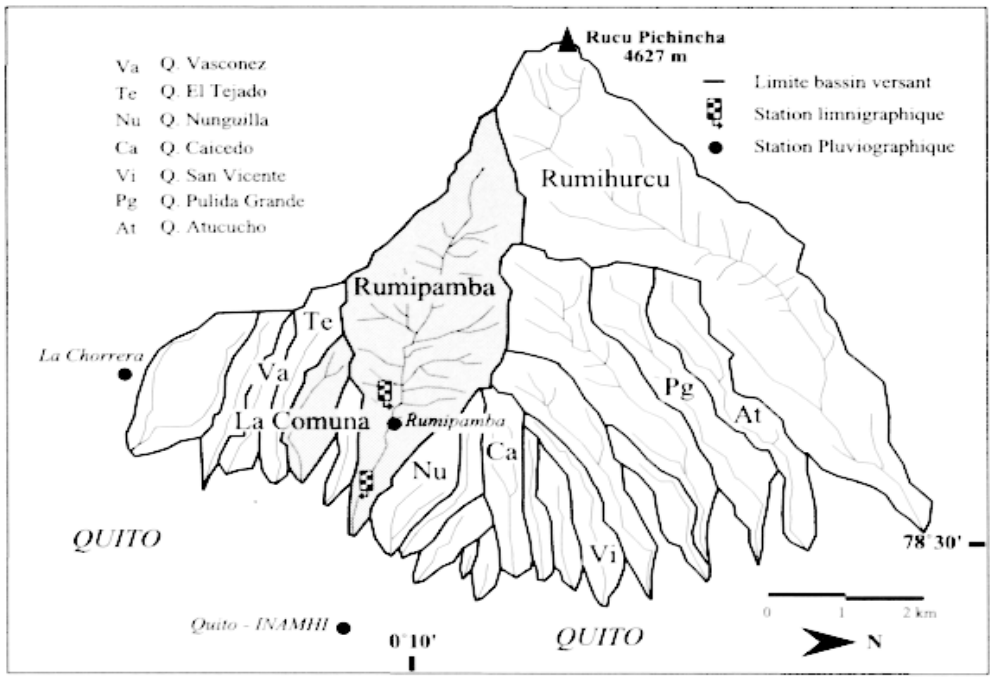

Fig. 2 : Quebradas du flanc nord-oriental du Pichincha - Réseau de mesures hydro-climatologiques

De nombreuses ravines, les quebradas, entaillent profondément les versants recouverts d'épaisses couches de cendres témoins des différentes éruptions du volcan. Elles individualisent des bassins souvent très allongés de quelques hectares à une dizaine $\mathrm{de} \mathrm{km}$ 'où les pentes dépassent fréquemment 20 à $30^{\circ}$. Sur le flanc oriental du Pichincha, plus 
d'une trentaine de quebradas débouchent ainsi directement dans le réseau de drainage de la ville et sont susceptibles de provoquer, lors des averses les plus intenses, des écoulements torrentiels fortement chargés.

Le 31 mars 1997, une coulée de boue chargée de blocs rocheux, de débris végétaux et de divers matériaux traversait le quartier de Ia Comuna au pied de la quebrada de I.a Comunidad (Fig. 2). Son passage dans ce quartier était à l'origine de très gros dégâts matériels et du décès de deux personnes.

La littérature sur les mouvements de terrain fait état de plusieurs systèmes d'appellation et les distinctions entre glissements boucux, glissements coulées, coulées boucuses, crues boueuses et écoulements hyper-concentrés sont parfois difficiles à mettre en évidence. Toutefois, compte tenu des concentrations en éléments solides de ces fluides et des phénomènes hydrauliques qui les caractérisent, le terme le plus approprié pour les qualifier semble être celui de lave torrentielle. Ce dernier désigne des écoulements boueux et rocailleux qui surviennent dans le lit des torrents de montagnes après de longues et fortes périodes de pluies. Flles ont l'aspect d'un fluide très visqueux, capable de transporter des blocs de plusieurs mètres de diamètre. Dès lors, la quantité de matériaux solides transportés et l'énergie développée lors de tels phénomènes sont beaucoup plus importantes que lors des écoulements torrentiels normaux (Meunier, 1991, Coussot, 1996 et Besson, 1996).

Après une description générale du phénomène et de ses conséquences, nous analyserons les principaux facteurs à l'origine de celui-ci. Finalement, nous replacerons cet événement dans le contexte historique des laves torrenticlles et des coulées boucuses observées depuis 1960 sur la zone métropolitaine de Quito.

\section{Description du phénomène et de ses conséquences}

Nous reprendrons ici les observations effectuées dès le lendemain de l'événement, en distinguant trois grands secteurs caractérisés par des mécanismes spécifiques de formations, transfert et/ou dépôts des laves torrentielles sur les flancs du Pichincha.

\subsection{DÉCI.ENCHEMEN'T IDE IA L.AVE TORRENTIFIILE:}

A la côte $3500 \mathrm{~m}$, on observe un glissement de terrain dans le fond de la quebrada (point 1, Fig. 3). Trente mètres cubes de sol ont glissé le long d'un plan structural à fort pendage orienté dans le sens même de la pente.

A quelques mètres du point de décrochement, ce glissement de terrain (probablement déjà peu visqueux) et le flux d'eau (venant de la partie haute de la quebrada) se mélangent de façon immédiate, avant de chuter presque à la verticale, une cinquantaine de mètres en dessous au niveau d'une cascade. Si, en amont du décrochement, la quebrada a gardé sa forme d'avant l'événement, il n'en va pas de même à l'aval où elle a été en grande partic dévastée. Les racines enchâssées entre des blocs de roche-mère totalement 


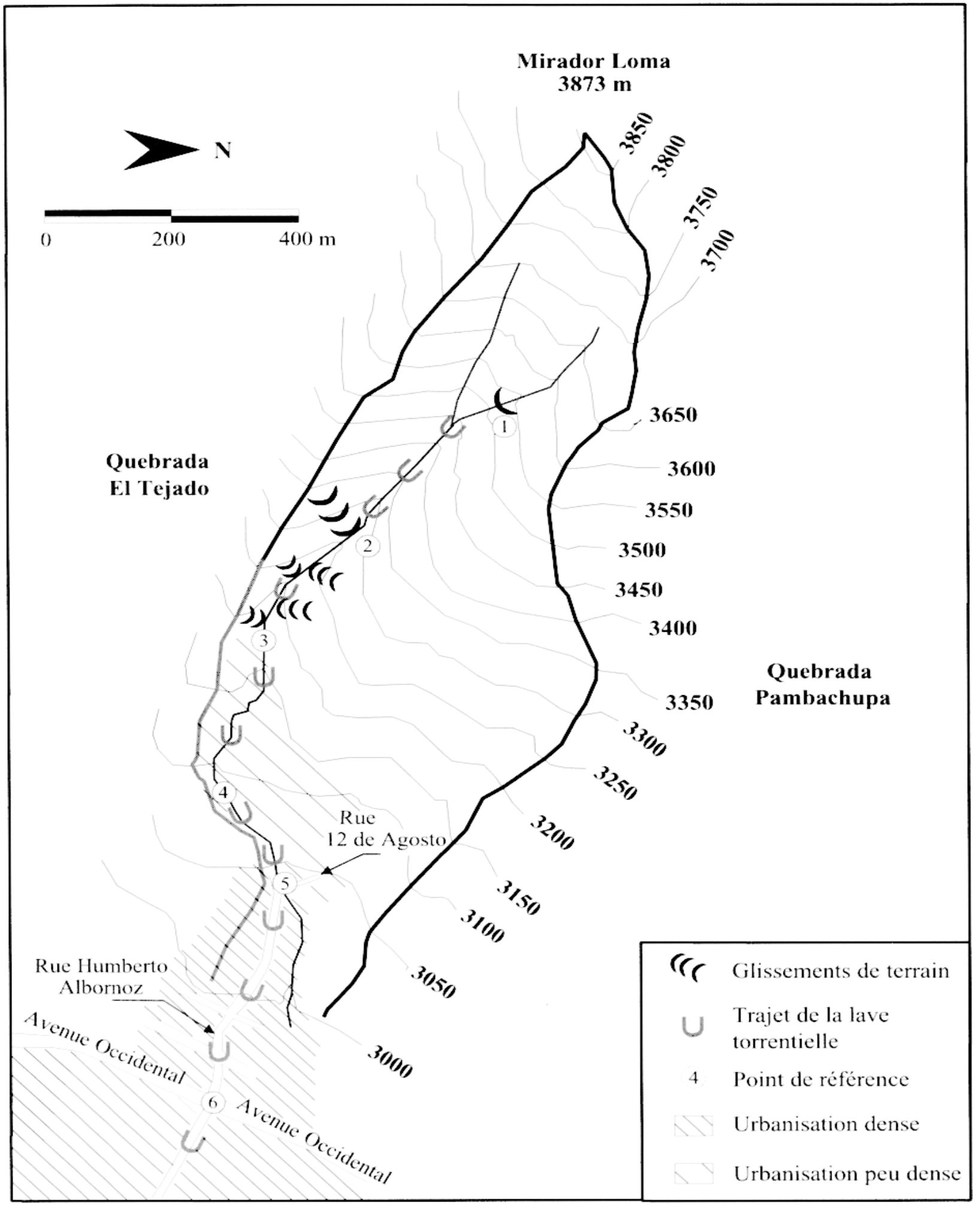

Fig. 3 : Bassin versant de La Comunidad - Localisation des différents mouvements de terrain

décapés témoignent de la violence de l'écoulement, qui sur 4 à $5 \mathrm{~m}$ de large a arraché arbres, arbustes et enlevé des pierres de grande taille. Certains blocs ont sans doute été projetés dans la quebrada, comme le prouvent les branches cassées ou arrachées plusieurs mètres au-dessus des traces laissées par la coulée boueuse elle-même. 
Quelques mètres en dessous de la cascade, et malgré des vitesses probablement supérieures à $10 \mathrm{~m} / \mathrm{s}$, la coulée se stabilise dans le cours (ici moins pentu), projetant dans chaque courbe ou à chaque ressaut de la boue à plusicurs mètres au-dessus du cours actuel du ruisseau. Aucune abrasion (le fond du lit étant constitué de roche-mère peu fracturée et peu altérée) ou érosion latérale importante n’a été observée sur cette partic du cours où seule la végétation des rives et du fond a été arrachée.

\subsection{APPORT EIT TRANSFERT DF MATÉRIEI.}

A partir de $3350 \mathrm{~m}$ d'altitude, le long du cours, une vingtaine de glissements de terrain latéraux plus ou moins importants, apportent des quantités substantielles de matériel avant, pendant et après le passage de la coulée boueuse (point 2, Fig. 3). Le glissement le plus important, situé sur le versant de rive droite de la quebrada, a environ 8 mètres de large et a emporté sur environ 80 mètres de long une épaisseur de $80 \mathrm{~cm}$ de sol, entraînant ainsi de 500 à $600 \mathrm{~m}^{3}$ de terre et rochers. En aval de cette zone, les observations de terrain montrent un accroissement net de l'intensité de l'érosion. Du fait de cet apport massif en matériel (et donc de l'augmentation probable de la viscosité de la coulée de boue), le fond, marqué par des ressauts successifs, a été par endroit très fortement entaillé (parfois sur plus de $2 \mathrm{~m}$ de profondeur).

Jusqu'à $50 \mathrm{~m}$ de la sortie de la quebrada, le schéma reste à peu près le même. Ces secteurs marqués par une très forte érosion du fond présentent ça et là des zones d'accumulation de grosses pierres (d'un volume souvent supérieur au $\mathrm{m}^{3}$ ). De profondes stries (dans le matériel tendre) et de nombreux éclats (sur les roches dures en place) marquent encore l'importance du transport solide et la violence de la coulée boueuse.

\subsection{PASSAGiF IDANS I.E.S SFC,THURS URBANISESS HI ACCUMUI.ATION DU MATERIFI.}

Au sortir de la partie encanyonnée, la quebrada s'élargit brutalement formant un vaste champ d'épandage (point 3, Fig. 3). Parallèlement, la pente du lit diminue fortement, permettant une importante perte de compétence de la coulée. Cette diminution des potentialités de transport se traduit par le dépôt de blocs de très grande taille (d'un volume supérieur au $\mathrm{m}^{\text {') }}$ recouverts d'une couche de boue de 1 à $2 \mathrm{~cm}$ (Photo 1). Les blocs de plus petite taille, arrêtés par la végétation arbustive, se sont déposés quelques centaines de mètres en aval, sur une zone pseudo-plane récemment déboisée. Compte tenu des pertes de compétence qu'elle a occasionnées, cette surface d'épandage a joué un rôle fondamental pour la protection de la zone habitée située à l'aval.

Très vite, la coulée qui s'était largement étalée en amont du quartier La Comuna, se concentre à nouveau suivant l'ancienne quebrada, remblayée au fil des années par les habitants du secteur pour construire la voie d'accès à la partie haute du quartier (point 4, Fig. 3). La force de l'écoulement entaille profondément le remblai, ouvrant un nouveau cours entre le talus et la rue, emportant ainsi une grosse partic du matériel accumulé ici. 


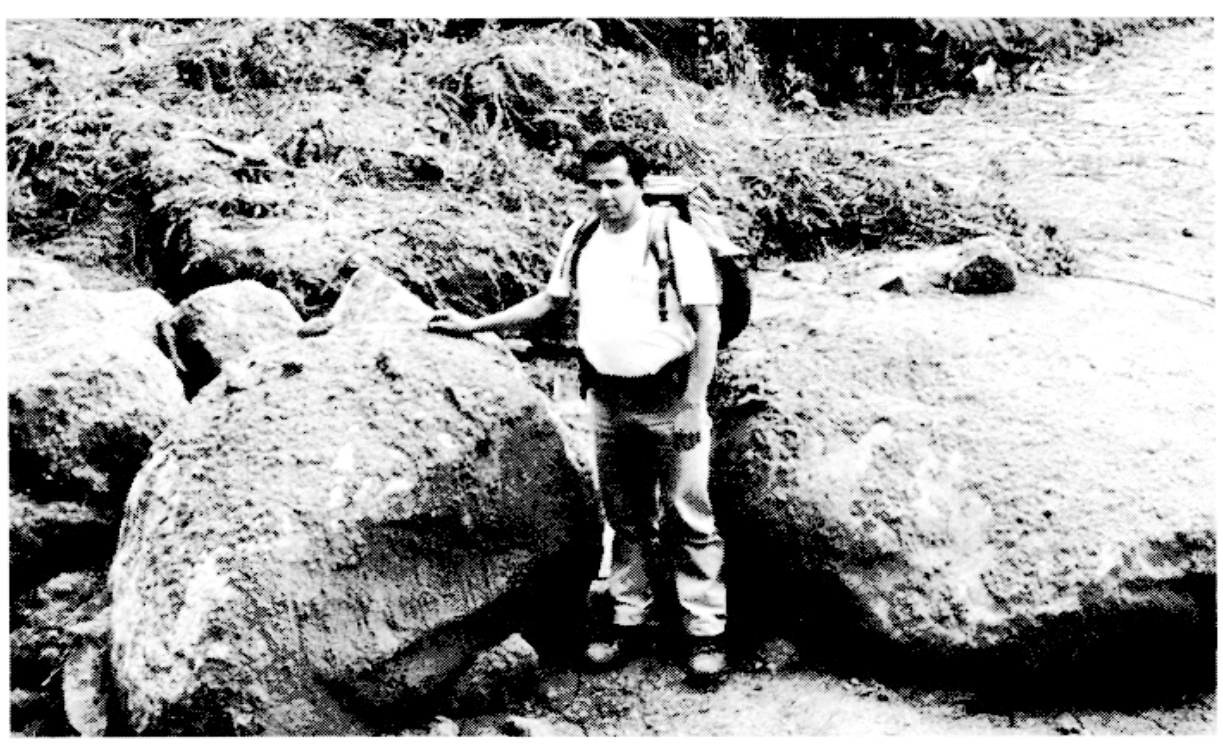

Photo 1 : Dépôts de pierres de grande taille à la sortie de la quebrada La Comunidad

Suivant l'ancien cours de la quebrada et la partie haute de la rue Humberto Albornoz, le flux rentre dans le quartier de I a Comuna et débouche au croisement avec la rue 12 de Agosto (point 5, Fig. 3). Au lieu de suivre le cours même de la quebrada (bouchée en totalité par la rue) la plus grosse partic du flux bifurque et suit la rue Albornoz en direction de l'avenue Occidental, détruisant au passage une maison située au croisement. Reprenant de la vitesse dans cette rue très en pente (plus de $20 \%$ ), la lave torrentielle, d'une épaisseur d'environ $1 \mathrm{~m}$, arrache tout sur son passage, tuant deux personnes et laissant à l'intérieur des habitations une grande quantité de boue (Photo 2).

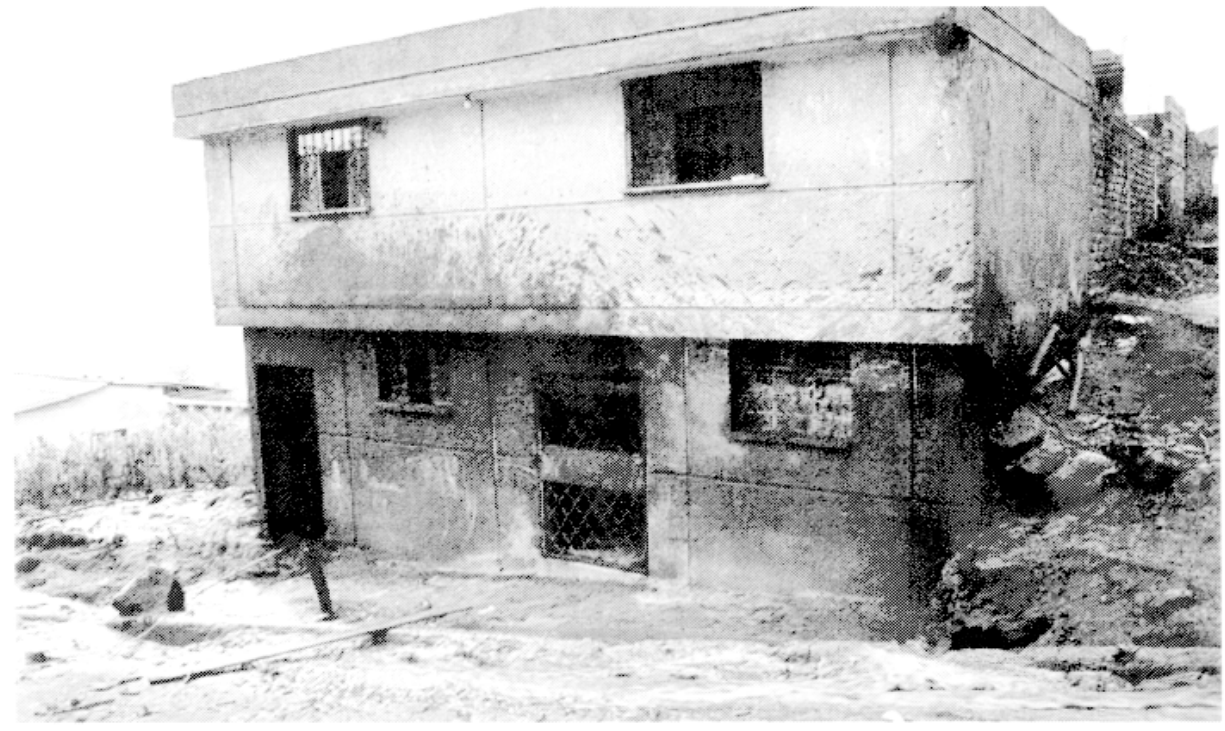

Photo 2 : Traces laissées par le passage de la lave torrentielle sur l'une des maisons du quartier

I a lave torrentielle, suivant la rue Humberto Albornoz, arrive ensuite sur l'avenue $O C$ cidental, où se dépose la plus grande partie du matéricl arraché sur la partie haute de la quebrada et dans la rue elle-méme. De $1 \mathrm{~m}$ à $1,5 \mathrm{~m}$ de boue s'accumule sur l'avenue, formant ici un point bas (point 6, Fïg. 3). A noter que l'avenue a joué ce jour-là le rôle d'un véritable bassin d'expansion permettant à la coulée de boue de s'étendre, en largeur, sur 
une bonne centaine de mètres. Ainsi, seule la partie la plus liquide a pu, par débordement, poursuivre son cours en dessous de l'Occidental, ne générant alors que des dégâts mincurs jusqu’à la Plaza Italia, un kilomètre plus bas.

\section{Principaux facteurs à l'origine du phénomène}

Le 31 mars 1997, un événement pluvieux exceptionnel touchair le versant oriental du volcan Pichincha. La conjonction de fortes précipitations (facteur naturel "déclenchant") et de conditions morpho-pédologiques spécifiques (facteurs naturels "permanents") allait être à l'origine de la lave torrentielle qui allait affecter la quebrada La Comunidad, générant d'importantes destructions dans le quartier de Ia Comuna (Fig. 2).

\subsection{Facteurs CimMATigues}

Il n'existe aucune station de mesures des précipitations sur le secteur même de l'étude (Fig. 2). Par contre, les informations collectées aux alentours (stations Rumipamba, La Chorrera et Quito-Inamhi situées dans un rayon de 3 kilomètres autour du bassin versant de La Comunidad), permettent d'avoir une connaissance assez précise de l'événement pluvieux ayant eu lieu ce jour là.

Le tableau suivant résume les principales caractéristiques des deux averses ayant eu lieu le 31 mars 1997 sur le secteur d'étude (Tab. 1) et montre bien la très grande variabilité spatiale des précipitations sur la ville de Quito (Pourrut et Leiva, 1989 et Bouvier et al., 1999).

\begin{tabular}{|c|c|c|c|c|c|c|c|c|c|}
\hline \multirow{2}{*}{\multicolumn{2}{|c|}{ Stations }} & \multirow{3}{*}{$\begin{array}{l}\text { Altitude } \\
\text { m }\end{array}$} & \multicolumn{3}{|c|}{ Première averse-P1 } & \multicolumn{3}{|c|}{ Deuxième averse-P2 } & \multirow[b]{2}{*}{$\begin{array}{l}\mathrm{P} \text { totale } \\
\mathrm{mm}\end{array}$} \\
\hline & & & $\mathrm{H}$ début & $\mathrm{H}$ fin & $\begin{array}{l}\mathrm{P} 1 \\
\mathrm{~mm}\end{array}$ & $\mathrm{H}$ début & $\mathrm{H}$ fin & $\begin{array}{l}\mathrm{P} 2 \\
\mathrm{~mm}\end{array}$ & \\
\hline Rumipamba & $\mathrm{Pg}$ & & 13 h 30 & $14 \mathrm{~h} 00$ & 3,5 & 15 h 15 & 17 h 05 & 14,0 & 17,5 \\
\hline Quito-Inamhi & $\mathrm{Pg}$ & 2789 & 13 h 50 & $14 \mathrm{~h} 20$ & 1,9 & 16 h 20 & $17 \mathrm{~h} 05$ & 3,3 & 5,2 \\
\hline La Chorrera & $\mathrm{Pm}$ & 3165 & - & - & - & - & - & - & 58,8 \\
\hline
\end{tabular}

Tab. 1 : Principales caractéristiques des 2 averses du 31 mars 1997 aux stations Rumipamba, Quito-Inamhi et La Chorrera (Pg : pluviographe ; Pm : pluviomètre)

Il est malheureusement impossible de reconstituer l'histoire de l'événement sur le secteur de La Comuna. Par contre sur la base de la valeur obtenue à la station la Chorrera $(58,8 \mathrm{~mm})$ et avec le modèle de répartition temporelle des pluies de la station Rumipamba, en première approximation, la pluie de la première averse est estimée à $12 \mathrm{~mm}$ et la seconde à $47 \mathrm{~mm}$. Avec une précipitation de $47 \mathrm{~mm}$ en 1 heure 50 minutes (soit $26 \mathrm{~mm} / \mathrm{h}$ ) à la Chorrera, la période de retour de l'événement est d'environ 50 ans (Ayabaca et al., 1996). 
Compte tenu des témoignages recueillis, il semblerait qu'une cellule convective extrêmement dynamique se soit formée sur le secteur concerné. Celle-ci serait ensuite restée bloquée au-dessus de La Comuna, comme le montre bien, la différence existant entre le début de la pluie à la station Rumipamba (15 h 15) et à la station Inamhi (16 h 20), pourtant relativement proches l'une de l'autre.

Deux stations de référence (Chorrera et Quito-Inamhi) permettent de comparer les précipitations antécédentes du mois de mars 1997 avec les moyennes mensuelles interannuelles (Tab. 2).

\begin{tabular}{|l|l|l|l|l|l|l|}
\hline Stations & P mars 1997 & -30 jours & -15 jours & -7 jours & -3 jours & P moyenne mars \\
\hline Chorrera & 231,0 & 172,2 & 147,5 & 40,3 & 30,9 & $149,9(1978 / 1993)$ \\
Rumipamba & 223,5 & 206,0 & 176,0 & 81,7 & 60,4 & non disponible \\
Inamhi & 167,7 & 162,0 & 117,8 & 21,3 & 17,8 & $135,4(1977 / 1993)$ \\
\hline
\end{tabular}

Tab. 2 : Précipitations antécédentes et moyennes mensuelles de trois stations du secteur - Événement du 31 mars 1997

Sans que l'on puisse le quantifier, il semble que les précipitations antécédentes aient pu jouer un rôle fondamental lors de l'événement du 31 mars 1997, en augmentant sensiblement l'humidité des sols et des formations superficielles du bassin versant. En effet, le tableau précédent (Tab. 2) montre que d'importantes précipitations avaient eu lieu sur le secteur dans les trois jours précédant la catastrophe $(60 \mathrm{~mm}$ à la Rumipamba et $31 \mathrm{~mm}$ à la Chorrera). De même, on peut noter que les précipitations durant les 15 jours précédant l'événement atteignent à la Chorrera $148 \mathrm{~mm}$ soit à peu près la valeur de la précipitation moyenne mensuelle de la station pour la période 1978/1993 $(150 \mathrm{~mm})$.

\subsection{FAC:JEURS MORPHOIOCILUES}

Si l'événement pluvieux de fréquence rare est l'un des principaux facteurs d'explication du phénomène, il semble que la morphologie même de la quebrada, et en particulier son profil en long, ait pu jouer un rôle fondamental dans la génération de ce type d'événement.

A partir d'un modèle numérique de terrain (pas $20 \mathrm{~m}$ ), nous avons extrait les profils en long de toutes les quebradas comprises entre le secteur de La (iasca et l'extrême nord de la ville de Quito (Fig. 4). La simple comparaison des différents profils est riche d'enseignements.

On peut ainsi différencier deux grands groupes de quebradas:

- Ie premier, très homogène, rassemble la plupart des petites quebradas, ayant unc superficie inféricure à $1,5 \mathrm{~km}$. Celles-ci se caractérisent par des profils en long à forte pente (en général supéricure à $30 \%$ ), au moins sur leur portion moyenne. Trois quebradas de ce groupe sont connues pour avoir généré des coulées de boue ayant provoqué des dommages importants dans les quartiers périphériques de la ville. Il s'agit des 


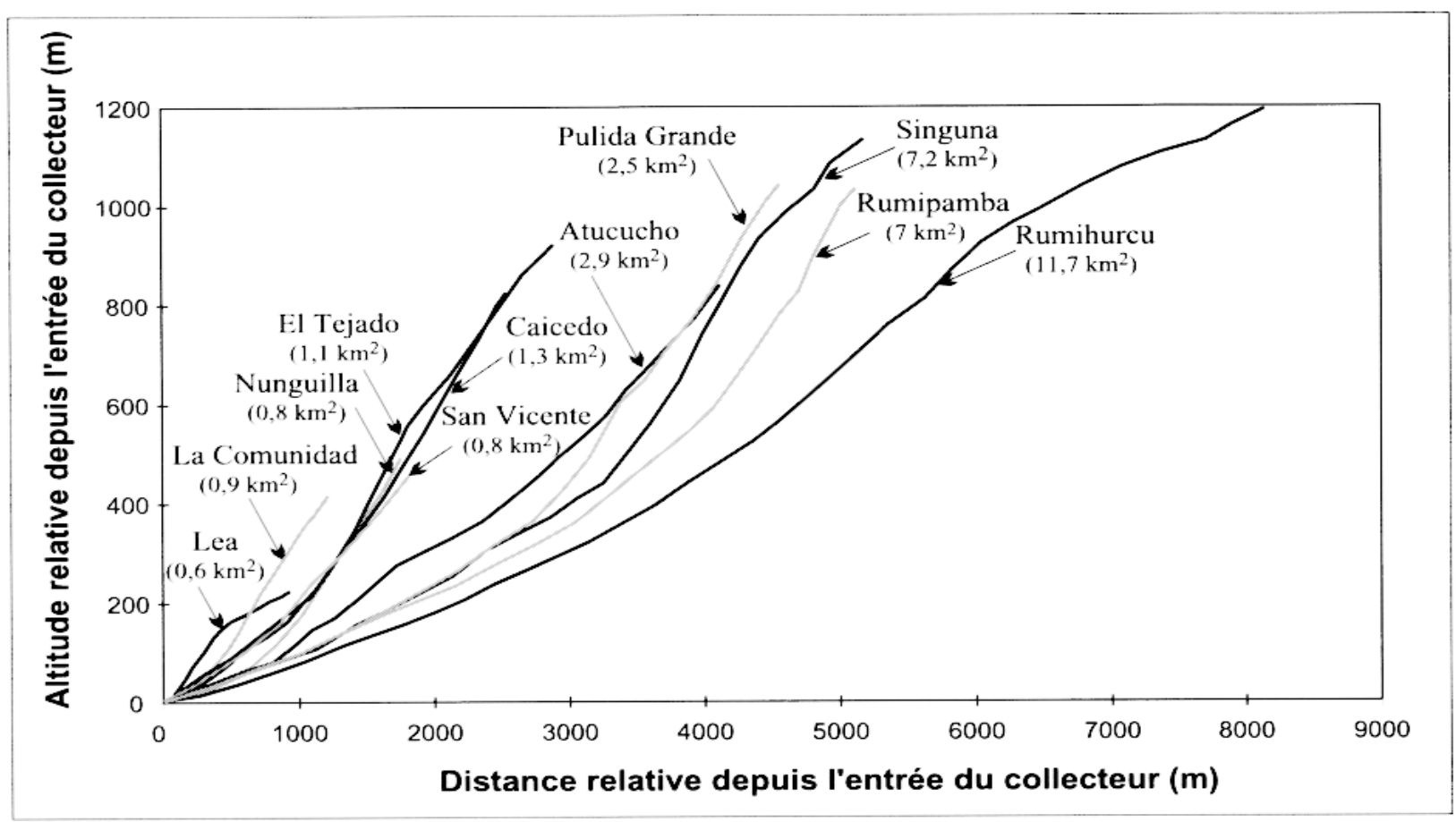

Fig. 4 : Profils en long des principales quebradas de la zone nord occidentale de Quito

quebradas La Comunidad (La Comuna-1997), El Tejado (La Gasca-1975) et I a I.ea qui malgré sa faible superficie $\left(0,6 \mathrm{~km}^{2}\right)$ a généré une coulée boueuse ayant recouvert (à la fin des années 1980) l'Aéroport International de Quito de plusieurs centimètres de boue, perturbant ainsi, durant plusieurs jours le trafic aérien.

- Le second, beaucoup moins homogène, rassemble les quebradas ayant une superficie supérieure à $1,5 \mathrm{~km}$. Celles-ci se caractérisent par des profils en long beaucoup moins pentus (compris entre 15 et $20 \%$ ), comme c'est le cas par exemple, des quebradas Rumihurcu et Rumipamba (les deux plus grandes du secteur d'étude).

I.es quebradas du premier groupe (à profils en long très pentus) sont bien évidemment plus sujettes à des événements du type de celui de La Comuna, que la quebrada Rumihurcu, moins pentue et plus encaissée du fait d'une évolution géomorphologique plus longue. Dès lors, il semble que les petites quebradas puissent être plus dangereuses que les grandes pour les quartiers situés en périphéric de la ville.

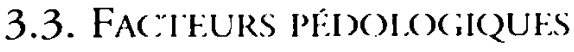

Les profils pédologiques suivants ont été observés sur les secteurs fortement affectés par les glissements de terrain :

- 0-40 cm - Horizon organique humifere (A1): Horizon noir, à texture limoneuse, bien structuré et caractérisé par un volume de vides important lié à l'activité biologique.

- 40-100 cm - Horizon organique humifere (A2) : Horizon marron foncé, à texture limono-sableuse présentant un faciès massif. 
- Contact brutal avec une dalle d'andésite sub-parallèle à la pente ou avec un horizon endurci d'altération de la roche mère en place, constituant un plan de glissement préférenticl.

I.es principales particularités de ces profils, typiques des versants à forte pente et du fond des quebradas des flancs du Pichincha, sont d'une part leur faible épaisseur (Poulenard, 1996, Jancau et al., 1997), et d'autre part leur contact brutal avec un plan de glissement préférentiel (Risser, 2000).

Afin de compléter l'étude, quelques analyses ont été effectuées sur des échantillons prélevés directement au-dessus du plan de glissement supposé. Ce niveau est composé d'une proportion importante $(\geq 50 \%)$ d'éléments et sables grossiers (particules $>0,2 \mathrm{~mm}$ ) enchâssés dans une matrice limoneuse (limon $\geq 35 \%$ ). Ces horizons semblent pouvoir se saturer très rapidement, les capacités de rétention à saturation et à $\mathrm{pF}_{2.5}$ (capacité au champ), étant particulièrement faibles (de l'ordre de $60 \%$ et $25 \%$ respectivement), et avoir des propensions particulières à franchir les limites de plasticité et de liquidité pour de faibles quantités d'eau. Ce dernier point semble confirmé par les mesures des limites d'Atterberg, (obtenues par la méthode de la boite de Casagrande), à savoir : limite de liquidité $=44 \%$, limite de plasticité $=33 \%$ et indice de plasticité $=11$.

Les caractéristiques de ces profils permettent d'avancer l'hypothèse suivante quant à la genèse des glissements. Des circulations d'eau entre la roche-mère imperméable (ou un horizon induré) et un sol peu épais fortement imbibé (voire saturé) par les très fortes précipitations (ayant eu lieu lors de l'événement et durant les 3 ou 4 jours le précédant), ont favorisé le glissement de quantités non négligeables de matériel liquéfié dans le lit de la quebrada.

\section{L'événement de la Comuna dans le contexte historique des laves torrentielles et coulées boueuses observées depuis 1960 à Quito}

Peltre (1989a et 1989b) a recensé dans les articles de presse plus de 300 événements hydro-morpho-climatiques responsables de 517 accidents ponctuels d'extension et de gravité diverses entre 1900 et 1988. Si les coulées boucuses et les laves torrentielles sont, globalement, les événements les moins fréquents, ce sont en général les plus destructeurs et les plus meurtriers. Ayant lieu en périphérie de la zone urbaine, mais pouvant largement toucher les infrastructures de la ville compte tenu de leur extension, ces phénomènes sont toujours liés au tracé actuel ou ancien des quebradas.

Entre 1960 et 1988, 24 événements de type cruc boueuse ou lave torrenticlle ont été recensés, dont 13 graves à très graves ont entraîné des dégâts matériels importants et/ou la perte de vies humaines. 


\subsection{EVENIMHNTS SIMILAIRIS: LA RAYA (1986) HI I A GASCA (1975)?}

Malheureusement, depuis 1960, seuls deux événements ont été étudiés et sont bien documentés :

- Ia Raya (23 janvier 1986) sur le bassin versant de la quebrada du méme nom, - I a Casca (26 février 1975) sur le bassin versant de la quebrada El Iejado

Dans le cas de la Raya (De Noni et al., 1988), il semble que le phénomène observé s'apparente plus à une crue boucuse (écoulement hyper-concentré) sur une zone en pleine mutation du fait de son urbanisation. En effet, un violent orage, très localisé, serait tombé sur le quarticr. $\mathrm{I}$ a forte érosion de secteurs très remaniés, associée au dysfonctionnement d'un réseau d'égouts en cours de réfection scrait à l'origine de cet événement. Ce dernier, sans gravité, n’a finalement perturbé que le trafic routier et inondé quelques rez-de-chaussée. Nulle part il n'est fait mention de vitesses d'écoulement importantes ou d'un phénomène particulièrement destructeur.

L'analyse de l'événement du 26 février 1975 à La Gasca (Feininger, 1976) paraît ici beaucoup plus intéressante. En effet, comme pour la Comuna, les descriptions dont nous disposons laissent à penser qu'il s'agissait d'une lave torrentielle.

Feininger (1976) parle lui aussi d'une précipitation exceptionnelle, extrémement localiséc sur le secteur de I a Gasca. La lave torrentielle, selon lui, aurait alors été provoquéc par une très forte crue ayant érodé le bas des versants de la quebrada, remobilisant le matériel stocké le long du cours. Toutefois, l'auteur mentionne trois faits importants également observés sur la quebrada Ia Comunidad, pouvant donner lieu à une explication différente du phénomène :

- Comme à la Comuna, aucune cruc destructrice n'a été observée sur les quebradas voisines. Bien que très localisée à l'échelle de la ville de Quito, l'extension des cellules pluvieuses liées à ce type d'événement dépasse toujours les deux kilomètres carrés (superficie approximative de l'ensemble des deux quebradas: El lejado et I a Comunidad). En conséquence, deux processus peuvent être ici distingués, d'une part, des laves torrentielles ayant une origine spécifique (La Casca, 1975 et La Comuna, 1997), et d'autre part des crues purement hydrologiques de faible amplitude observables au pied du Pichincha lors de chaque événement pluvieux de fréquence rare (Ayabaca et Perrin, 1996).

- De même, l'auteur mentionne l'existence, sur le cours de la quebrada, d'une cascade de quelques $40 \mathrm{~m}$ de haut et s'étonne de la très faible érosion du lit en amont de celle-ci, avançant l'hypothèse d'une protection plus efficace de la végétation sur ce tronçon du cours. Nous avons observé, à La Comuna, le même type de phénomène, l'expliquant par le glissement et la rapide liquéfaction d'une quantité réduite de matériel ayant par la suite chuté sur $50 \mathrm{~m}$, quasiment à la verticale, acquérant ainsi une énergie cinćtique suffisante pour tout détruire en aval.

- Finalement Feininger note l'existence, tout au long du cours de la quebrada, de plusieurs glissements de terrains latéraux ayant apporté des quantités non négligeables de matéricl (plusieurs centaines de $\mathrm{m}^{\prime}$ ). 
En conséquence, il semble que deux phénomènes similaires (une lave torrentielle), ayant a priori les mêmes causes, et globalement les mêmes conséquences ont été observés le 26 février 1975 à I a Casca et le 31 mars 1997 à L Comuna. Par contre, les destructions ayant touché la ville de Quito ont été beaucoup plus importantes lors de l'événement de La (iasca que lors de celui de la Comuna. Doit-on en conclure que le premier, pour des raisons aujourd'hui inconnues, aurait été plus important que le second?

En 1976, Feininger mettait en garde les autorités sur les conséquences que pourrait avoir la construction de l'Avenue ()cidental sur la stabilité des sols et des formations superficielles du pied du Pichincha. ()r, 20 ans après, suite à l'événement de La Comuna, il semble que l'Avenue Occidental (construite à la fin des années 1970) ait cette fois protégé la ville d'un désastre peut-être comparable à celui de 1975. L'avenue a joué ici le rôle d'un véritable bassin " tampon ". Seule la partic la plus liquide a pu, par débordement, poursuivre son cours en aval, ne générant ensuite que des dégâts mineurs. F́n conséquence, cet ouvrage pourrait être, au moins sur certains tronçons, la protection la plus efficace dont disposerait actuellement Quito face aux laves torrentielles.

\subsection{EVENHMINT MINHLR : RLMMPAMBA (1998)?}

Des laves torrentielles provenant essentiellement des affluents à fortes pentes sont observées périodiquement dans le lit des grandes quebradas. Ces événements, rarement catastrophiques, ne génèrent quasiment jamais d'accidents au niveau des zones urbaines et ne sont donc pas recensés par la presse locale. Le 27 février 1998, un événement de ce type détruisait partiellement l'une des quebradas affluentes située en rive gauche de la quebrada Rumipamba, ne perturbant que durant quelques jours la distribution d'eau potable suite à l'envasement d'une captation.

I.es observations de terrain, menées dès le lendemain montraient clairement qu'un événement similaire à celui de la Comuna avait touché cette quebrada très en pente. Comme nous l'avions vu un an plus tôt, une petite quantité de matériel (estimée ici à $60 \mathrm{~m}^{\prime}$ ) avait brutalement glissé, s'était rapidement liquéfiée avant de chuter dans unc cascade pseudo verticale d'une soixantaine de mètres de haut.

Le lit de la quebrada, très large au pied de la cascade, avait été totalement remanié. I'importants phénomènes d'érosion latérale (ayant sur une centaine de mètres de long et plus de vingt de large décapé une couche d'un mètre de sol ou de formations superficielles) pouvaient y être observés. Plus de $2000 \mathrm{~m}^{i}$ de matéricl divers avaient été emportés formant ainsi la plus grosse partie de la coulée de boue. La lave torrentielle avait par la suite dévalé l'ensemble du cours de la quebrada arrachant la végétation présente au fond du lit, sans toutefois augmenter de façon significative son volume. A la confluence avec la quebrada principale, une très forte diminution de la compétence du flux liée à une diminution de la pente générale du cours, permettait le dépôt des blocs de grande taille et des éléments les plus grossiers. I ans le même temps l’important débit liquide provenant de la quebrada principale allait permettre une dilution du matériel solide en suspension, diminuant ainsi fortement la densité et donc la capacité érosive de la lave torrenticlle. 
Celle-ci se transformait peu à peu en écoulement hyper-concentré et poursuivait, sans générer de destruction majeure, son cours jusqu'à la partie basse et urbanisée de la quebrada. Du fait de l'encaissement du lit, cette crue boueuse avait pu transiter sans problème, avant d'être évacuée par le système d'égouts de la ville de Quito.

L.e 27 février 1998, un événement pluvieux exceptionncl touchait de nouveau les flancs du Pichincha. Avec une précipitation de $45,9 \mathrm{~mm}$ en 1 heure 15 minutes (soit $36,7 \mathrm{~mm} / \mathrm{h}$ ), enregistrée à la station Rumipamba, la période de retour de cet événement était estimée à environ 80 ans. Par contre, les précipitations antécédentes, importantes durant les 15 jours antérieurs à l'événement $(129 \mathrm{~mm})$, ont été nulles durant les trois jours le précédant.

Bien que les sols des quebradas Rumipamba et La Comunidad soient en tout point comparables, aucun glissement de terrain n'a pu être répertorié sur les versants de la quebrada Rumipamba. L'absence de pluie dans les trois jours précédant l'événement pourrait être responsable de ce fait. Sur la quasi totalité du bassin, les sols ont pu absorber la précipitation du 27 février 1998, sans se déstabiliser ni dépasser les limites de plasticité ou liquidité. Dès lors, l'absence d'apport en matériel supplémentaire le long du parcours de la coulée de boue a probablement joué un rôle fondamental dans l'évolution de la lave torrentielle et limité, de façon significative, l'ampleur du phénomène observé ce jour-là.

Plusieurs événements de ce type peuvent être observés chaque année sur les différentes quebradas du Pichincha. Toutefois, l'exemple de la Rumipamba montre bien qu'il faut qu'un ensemble de facteurs géomorphologiques, pédologiques, climatologiques et hydrologiques s'associent pour que ce type d'événements se transforme en véritable catastrophe comme celle que nous avons pu observer sur le bassin versant de La Comunidad.

\section{Conclusion}

En guise de conclusion, un certain nombre de points doivent être mis en exergue. Les parties haute et moyenne des bassins versants de I a Comunidad et de la Rumipamba n'ont pas été anthropisées et sont parfaitement conservées pour l'instant. Les différents mouvements de terrain mis en évidence sur les secteurs critiques des bassins et ayant généré des coulées boueuses sont d'origine purement naturelle et n'ont aucun rapport avec une quelconque activité anthropique (déforestation, surpâturage ou canaux de drainage). Ainsi :

- La conjonction d'une période particulièrement humide et d'un événement pluvieux exceptionnel semble être, dans tous les cas de figure, le principal facteur de formation des laves torrentielles.

- Les très fortes pentes observables au niveau même des cours, et en parriculier à proximité des zones de déclenchement, permettent à quelques dizaines de mètres cubes initialement mis en mouvement d'acquérir une vitesse et une compétence importantes. A ce titre, les petites quebradas, souvent très pentues, seraient plus susceptibles de voir leurs cours détruits par des laves torrentielles que les grandes. 
- La présence d'une grande quantité de matéricl au niveau même du cours, issue de glissements de terrain ayant eu lieu sur les flancs des quebradas, permet à la lave torrentielle d'augmenter significativement son volume, mais aussi sa viscosité (et donc sa capacité érosive). Il en va de même pour l'ensemble du matériel formant le fond de la quebrada, qu'il s'agisse de pierres, de colluvions ou plus simplement de la végétation arrachée par la lave torrentielle clle-même.

Si les grands mécanismes de formations des laves torrentielles à Quito semblent connus, d'importantes zones d'ombre subsistent. En effet, les processus de transformation d'un glissement de terrain ou d'un glissement coulée en une lave torrentielle sont encore très mal connus et pourraient faire l'objet d'une étude spécifique. De même, les analyses rhéologiques effectuées par le Cemagref de Grenoble (Richard, 1997) d'échantillons prélevés après le passage de la lave torrentielle de I a Comuna montrent que le fluide correspondant ne peut pas obéir à la loi de comportement de Herschel-Bulckley identifiée comme modèle rhéologique des laves torrenticlles boueuses des Alpes. Ceci n’est pas véritablement une surprise compte tenu de la nature volcanique du matériau mobilisé, dans la mesure où les lahars sont en général caractérisés par des écoulements granulaires ne répondant pas à ce modèle. Dès lors, l'identification de la loi de comportement de ces mélanges à base de cendres volcaniques reste un important sujet de recherche. Dans ce cadre, Quito pourrait être considéré comme un site intéressant d'études.

Si des phénomènes naturels sont, dans la plupart des cas, le détonateur de l'accident, ils n'en expliquent que partiellement les conséquences. L'activité humaine, responsable de la vulnérabilité des quartiers périphériques de la ville, joue un rôle fondamental dans la genèse des désastres. Ainsi :

- L'occupation urbaine de terrains inadaptés (fortes pentes, instabilité des sols, lits de quebradas...) et menacés par différents alćas naturels (pluies intenses, crues, coulées de boue, glissements de terrain...) constitue le facteur prépondérant de vulnérabilité des zones périurbaines de la ville de Quito. La diminution des risques devrait passer, avant tout, par un contrôle strict de la dynamique urbaine et de l'utilisation des sols de ces secteurs.

- les caractéristiques physiques et techniques de l'urbanisation sont souvent des facteurs aggravants, qu'il s'agisse du remblayage des quebradas, de la construction de rues parallèles à la pente ou du déboisement. Ces pratiques depuis longtemps connues, se sont malheureusement généralisées à l'échelle de la ville dans le cadre de la consolidation urbaine. I.es quebradas ouvertes sont devenues rares, certains secteurs déboisés ont été durablement construits et aujourd'hui, la géométric du tissu urbain n'est plus modifiable. Ces facteurs de vulnérabilité sont done devenus permanents.

- Les conditions socio-économiques forment un dernier groupe de facteurs. Elles expliquent en partic la dynamique d'occupation et se traduisent par l'insuffisante préparation d'une population, souvent désemparée quand survient l'accident.

Même si cela est souvent le cas, l'activité anthropique n'est pas toujours à l'origine des désastres dits " naturels". Activité humaine ou pas, des événements du type de celui de I a Comuna continueront à avoir lieu au pied du Pichincha. Sans politique durable et efficace de prévention et de protection, ces accidents pourront toujours se traduire, a l'échelle d'un quartier, par des destructions importantes voire la perte de vies humaines. 


\section{Remerciements}

Nous remercions nos partenaires du programme SISHIL AD (Sistema de Pronostico Hidrologico de las Laderas del Pichincha), l'EMAAP-Q (Empresa Municipal de Alcantarrillado y Agua Potable de Quito) et l'INAMHI (Instituto Nacional de Meteologia e Hidrologia) pour l'appui logistique et financier à ce projet. Nous remercions également le Programme National sur la Prévision et la Prévention des Risques Naturels (PNRN) de l'Institut National des Sciences de I'Univers (INSU, France) qui contribuc au financement du programme SISHILAD depuis 1996, ainsi que le Cemagref de Grenoble pour l'aide qu'il nous a apportée, en envoyant à Quito l'un de ses chercheurs et en permettant l'analyse de nombreux échantillons.

\section{Bibliographie}

Ayabaca E., Cru\% F., Gumirrey C., 1996. - Curvas Intensidad-Duración-Frecuencia de las principales estaciones pluviográficas de Quito. Rapport SISHILAD, EMAAP-Q / INAMHI / ORSTOM, 19 p.

Ayabac a E., Plikrin J.L., 1996. - Primer año de mediciones en la red hidrometeorológica de las Laderas del I'ichincha. Rapport SISHIL.AD, EMAAP-Q/INAMHI / ORSTOM, 26 p.

BtSSON L. (1996). - L.es risques naturels en montagne. Traitement, prévention, surveillance. Artès - Publialp, 438 p.

Bolvitir C., ayabacia F., Plrrin J.L., Cru\% F., Fourc ad de B., Rosario S., Carrfra l., 1999. Variabilités temporclle et spatiale des averses en milieu andin : Excmple de la ville de Quito (Équateur). Rev. Géog. Alpine, n 3, Tome 87, 51-65.

Cousso1 P., 1996. - Les laves torrenticlles - Connaissances à l'usage du praticien. Série Études, Equipements pour l'Eau et l'Environnement, n² 23, Cemagref, $177 \mathrm{p}$.

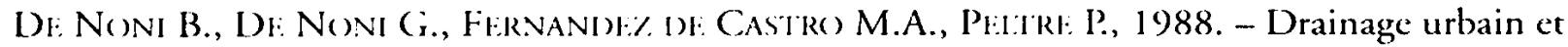
accidents climatiques à (Quito (Équateur) : Analyse d'un cas récent de crue boucuse. Cahier des Sciences Humaines, Vol. 24, $\mathrm{n}^{\circ}$ 2, ORSTOM, Paris, 225-249.

Fianincit: T., 1976. - El flujo de escombros en La Gasca: Un informe cientifico. Boletin de la Sección National de Ecuador, n 5-6, IPGH, 7 p.

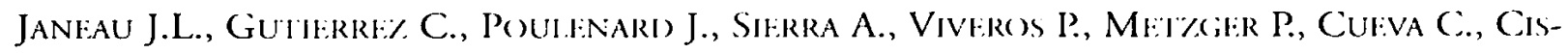
NFROS F., 1997. - Primeros resultados - Area de Edafología. Rapport SISHILAD, EMAAP-Q / INAMHI / ORSTOM, $222 \mathrm{p}$.

MFUnifR M., 1991. - Eléments d'hydraulique torrenticlle. Série Études Montagne nº 1, Cemagref, $278 \mathrm{p}$.

PHiTRI P., 1989a. - Quebradas y riesgos naturales en Quito, periodo 1900-1988. Estudios de (jeografía, Vol. 2, 'Riesgos naturales en Quito - Lahares, aluviones y derrumbes del Pichincha y del Cotopaxi, Eds. Corporación Editora Nacional. Colegio de Geógrafos del Ecuador, 45-91.

PEITRR: P., 1989b. - Les accidents du drainage urbain à travers la presse : Quito (Équateur) 1900)1986.Tropiques - Licux et Liens, Ed. ORSTOM, 318-334.

PoUll:NARI) J., 1996. - Caractérisation et classification de sols dérivés de cendres volcaniques récentes (Volcan Pichincha - Équateur). Mémoire de DEA - Sciences du sol. Insticut National Agronomique Paris-Grignon, 52 p. + Ann.

Pourrut P., Llisva I., 1989. - Las lluvias de Quito : características generales, benificios y problemática. Estudios de Geografía, Vol. 2, 'Riesgos naturales en Quito - Lahares, aluviones y derrum- 
bes del lichincha y del Cotopaxi', Eds. Corporación Editora Nacional -.Colegio de Gégrafos del Fcuador, 33-44.

RICHARI) I)., 1997. - Compte rendu de mission à Quito (Équateur). Rapport ORSTOM / Cemagref, $14 \mathrm{p}$.

RIsst:R V., 2000. - Mouvements de terrain sur les versants orientaux du volcan Rucu Pichincha (Quito - Equateur). Rapport SISHILAD), IMAAP-Q / INAMHI / ORSIOM, 87 p.

Résumé : I. 31 mars 1997 , une lave torrenticllc affectait gravement la quebrada 1 a Comunidad. Des études morpho-bydro-climatiçues menées à la suite de cet accident ont montré que si l'événement pluvieux de fréquence rare étaic l'un des facteurs principaux d'explication du phénoméne, il apparaissait clairement que la morphologie méme des quebradas, ct en parriculier leur profil en long, pouvait jouer un rolc fondamental dans la génération de ceux-ci. De méme, il semble que les petites quebradas soient plus sujettes à ce type d'événements, les rendant ainsi plus dangereuses que les grandes pour les quarticrs sirués en périphérie de la ville.

Mots clés: Laves torrentielles, risques naturels, Quito, Équateur, Andes

\begin{abstract}
The $31^{\text {st }}$ of March 1997 a debris flow seriously affected the quebrada La (o)munidad and. Morpho-hydro-climatic studies done after this event showed that extreme rainfall storms was the main factor to explain these phenomenons, it is evident that the morphology of the catchment, and particularly the longitudinal profile, played a fundamental part in the gencration of debris flows. These events secm preferentially take place in the small catchments, therefore they can be considered as more dangerous than the big ones for the town suburban zones.
\end{abstract}

Keywords: Debris flows, natural hazards, Quito, Ecuador, Andes 